\title{
Diseño y Propiedades Psicométricas de un Cuestionario de Opinión sobre la Atención a la Diversidad (CUADIVER)
}

\author{
Design and Psychometric Properties of an Opinion Questionnaire \\ about Attention Given to Diversity (CUADIVER)
}

\author{
José Domínguez Alonso*, Antonio López Castedo \& Margarita Pino Juste \\ Universidad de Vigo, Vigo, Espanha
}

\begin{abstract}
Resumen
El presente artículo muestra el proceso seguido para diseñar e identificar las propiedades psicométricas de un cuestionario para evaluar la atención a la diversidad en centros educativos de primaria (CUADIVER), construido a partir de una metodología mixta. La primera fase de corte cualitativo (grupos focales) revela en su discurso (Aquad 5.0) un predominio de aspectos desfavorables (63.8\%) frente a los favorables (36.2\%) en la atención al alumnado diverso. En la fase cuantitativa, participaron 140 jefes de los departamentos de orientación de educación primaria, lo que representa un $61,14 \%$ de la población total $(N=229)$. Para su fiabilidad se calculó el Alfa de Cronbach obteniendo un índice muy aceptable .92 y el Método de las dos Mitades que presenta una correlación de .91 (ítems pares) y .82 (ítems impares). La solución factorial reprodujo la distribución de los ítems del cuestionario en el marco teórico, práctico y valorativo. Los resultados legitiman el CUADIVER como un instrumento de prestaciones psicométricas muy satisfactorias, que unido a sus propiedades de comprensibilidad y aceptación, lo convierten en una herramienta eficaz en la exploración y valoración de la atención a la diversidad en los centros educativos.
\end{abstract}

Palabras clave: Diversidad en Educación, métodos de investigación, cuestionarios.

\begin{abstract}
This article shows the process taken to design and identify the psychometric properties of a questionnaire (CUADIVER) developed from a mixed methodology to evaluate the situation of the attention given to diversity in primary education centers. In the first phase of the qualitative research the focal group shows us in its speech (Aquad 5.0) the predominance of unfavorable aspects (63.8\%) against favorable ones (36.2\%) in the attention given to diverse students. In the quantitative phase, 140 heads of orientation departments of primary education participated in the study, representing $61.14 \%$ of the total population $(N=229)$. Cronbach Alpha was calculated for its reliability obtaining a very acceptable index (.92). The Method of Two Halves was also applied and presented a correlation of .91 (even items) and .82 (odd items). The factorial solution reproduced the distribution of the items in theoretical, practical and assessment frameworks. The results legitimize CUADIVER as an instrument of very satisfactory psychometric benefits that joined to its properties of understandability and acceptance turns it into an effective tool in the development and assessment of attention given to diversity in education centers and schools.
\end{abstract}

Keywords: Institutional diversity, research methodology, questionnaires.

Cabe pensar que, en pleno siglo XXI, ninguna sociedad puede estar moralmente legitimada sino dedica su máximo esfuerzo a la atención de las personas o colectivos desfavorecidos. A nivel social, existe la convicción de que es necesario mejorar la atención a la diversidad de la población, pero también es una condición imprescindible de los sistemas educativos el ofrecer respuestas de calidad al conjunto de todo su alumnado (Casanova,

\footnotetext{
* Endereço para correspondência: Departamento de Análise e Intervención Psicosocioeducativa, Facultade de Ciencias da Educación de Ourense, Universidad de Vigo, Campus Universitario As Lagoas, Av. Castelao, s/n, Ourense, Espanha 32004.E-mail: jdalonso@edu.xunta.es, alopez@uvigo.es e mpino@uvigo.es
}

1999; Domínguez, 2009). Así pues, la relación existente entre una sociedad (en continuo proceso de cambio) y la enseñanza obligatoria (inmersa en continuas reformas legislativas), requiere constantes readaptaciones en los sistemas educativos para tratar de prevenir (Justicia et al., 2006), compensar o eliminar las desigualdades cada vez más presentes en la población. Una educación para todos que conjugue la excelencia y la equidad desde la diversidad es la alternativa actual a la desigualdad y la exclusión social (Jiménez \& González, 2010).

A día de hoy, la atención a la diversidad en el tramo obligatorio de la educación en los centros de enseñanza, es uno de los temas que más discusiones ideológicas y reflexiones pedagógicas crea en el marco educativo (Essomba, 
2003). Este hecho nos lleva a reflexionar desde la teoría y a analizar desde la práctica como están respondiendo los sistemas educativos al reto de favorecer el desarrollo integral de las personas a través de una educación inclusiva que pretende respetar la diversidad y compensar las desigualdades a partir de las necesidades, intereses y aptitudes de la población de referencia. Así pues, responder a la diversidad desde contextos educativos inclusivos representa "uno de los aspectos más delicados en el acontecer del actual sistema educativo" (Marchena, 2002, p. 2).

Los esfuerzos por llevar a la práctica los principios básicos de la atención a la diversidad (normalización, inclusión, equidad, igualdad de oportunidades, no discriminación, flexibilidad, accesibilidad, interculturalidad, promoción de la convivencia, autonomía de los centros y participación de toda la comunidad) no están exentos de problemas, constituyendo uno de los principales retos de las políticas nacionales e internacionales en materia educativa. No obstante, su creciente interés ha ayudado a percibir y apreciar que la diversidad es un estado natural, no un problema. En consecuencia, considerado el cuestionario una herramienta muy útil para describir de manera rápida y oportuna tendencias, frecuencias de opiniones y actitudes de una población, así como para conocer cómo se ha extendido y distribuido un fenómeno, ha hecho que, ante la escasez de instrumentos de supervisión de los sistemas educativos en el reto de ofrecer oportunidades de aprendizaje a todo el alumnado, nos lleve a confeccionar un cuestionario sobre la atención a la diversidad del alumnado en los centros educativos.

En el presente trabajo se presenta un instrumento, el cuestionario de opinión sobre la atención a la diversidad (CUADIVER), realizado utilizando un enfoque cualitativo (grupos focales) en un primer acercamiento a la realidad diaria de los centros de enseñanza para, posteriormente complementar y confirmar los datos obtenidos con una metodología de corte cuantitativo, con la única intención de obtener un conocimiento más profundo y completo de la problemática asociada a la atención del alumnado con necesidades educativas específicas. Nuestro principal objetivo es el diseño y posterior identificación de las propiedades psicométricas de un instrumento útil para su empleo en la evaluación e intervención psicoeducativa en el campo de la atención a la diversidad.

\section{Método}

Aunque, tradicionalmente se prefería la utilización por separado de técnicas cualitativas y cuantitativas, la tendencia actual es considerar que ambas deben combinarse proporcionalmente dentro de un marco común en la investigación empírica (Land, 2005). En la misma línea, Rubio y Varas (1999, p. 224) sostienen que "cuanto más amplia y plurimetodológica sea la investigación más posibilidades se tienen de conocer las diferentes dimensiones de una realidad siempre compleja e inabarcable" como es la educativa. A ello se debe añadir la gran revolución cualitativa de la última década que demuestra el interés de la comunidad científica por estas técnicas (Nicolaci da Costa, 2007).

Los incesantes cambios, problemas y fenómenos a los que tienen que enfrentarse los centros educativos son tan complejos y diversos que la utilización de un solo enfoque es insuficiente para lidiar con esta complejidad. Por ello, se requiere de métodos mixtos (Creswell, 2009) consecuentes con la especificidad de cada nivel de la realidad de referencia, lo que implica una coherencia entre la naturaleza del objeto de conocimiento y la forma para conseguirlo.

En función del marco teórico, la realidad escolar y el objetivo anteriormente expuesto, el propósito metodológico no es reemplazar la investigación cualitativa ni la cuantitativa, sino utilizar los puntos fuertes de ambas (Collins, Onwuegbuzie, \& Sutton, 2006) para la elaboración de un cuestionario:

- Enriquecimiento de la muestra.

- Mayor fidelidad del instrumento (certificando que este sea adecuado y útil).

- Integridad del tratamiento o intervención (asegurando su confiabilidad).

- Optimizar significados (facilitando mayor perspectiva de los datos, consolidando interpretaciones y la utilidad de los descubrimientos).

Así pues, el punto de partida es la exploración de la temática con diferentes grupos de profesorado en los centros educativos para posteriormente expandir el entendimiento de la problemática a muestras mayores y poder realizar generalizaciones a la población. Se utiliza un diseño exploratorio secuencial -DEXPLOS- (Hernández \& Mendoza, 2008) en tres etapas: (a) recabar datos cualitativos (grupos focales) y analizarlos (Aquad 5.0); (b) utilizar los resultados para construir un instrumento de medida cuantitativo (cuestionario CUADIVER); (c) administrar el instrumento a una muestra probabilística para validarlo.

Aunque la tendencia más generalizada en la actualidad educativa sea ver juntas las dos metodologías, esta complementariedad no está exenta de dificultades, tanto en su aplicación como en su interpretación (Stake, 1998). No obstante, su combinación va ha proporcionar un mayor beneficio a la producción de conocimientos (Bisquerra, 2000). La postura mixta (Axinn \& Pearce, 2006; SlonimNevo \& Nevo, 2009) es la que más ha crecido en los últimos años y será la que guiará el diseño en la elaboración del cuestionario (Figura 1).

\section{Participantes}

La primera fase de corte cualitativo (Boeije, 2009) se desarrolló a través de grupos focales (Barbour, 2007), durante cinco cursos académicos en centros de educación infantil y primaria. Los profesores participantes variaban en cada curso académico manteniendo la misma estructura y el mismo coordinador. En total han existido cinco grupos focales y han participado veinticinco profesores, un asesor del centro de recursos, tres directores, tres jefes de departamento de orientación y un coordinador. 


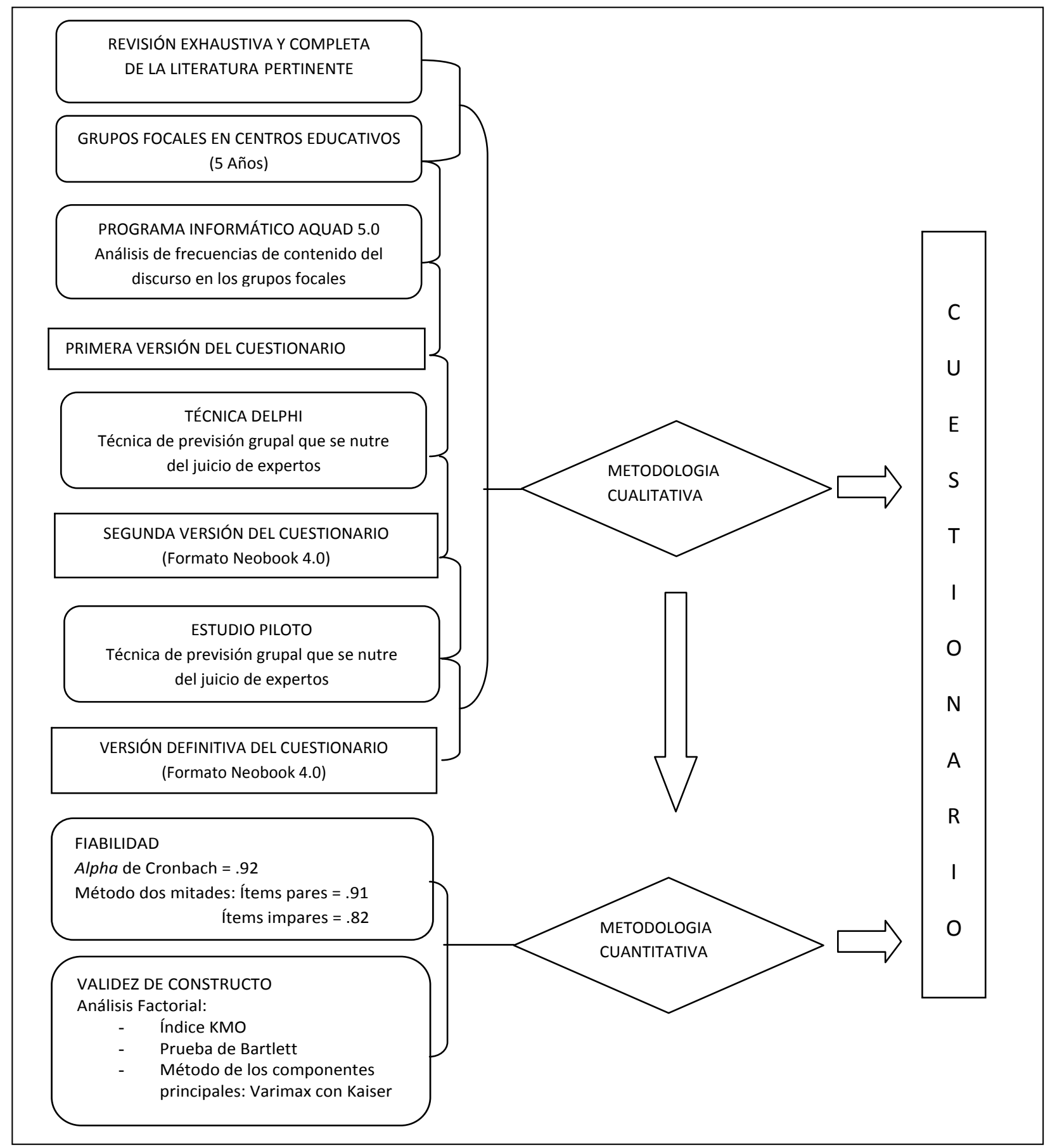

Figura 1. Esquema del proceso en la elaboración del cuestionario. Nota. Elaboración propia.

En la parte cuantitativa, asentándose en el principio de equiprobabilidad (todos los individuos tienen las mismas probabilidades de formar parte de la muestra) participaron 140 jefes de los departamentos de orientación de educación primaria en la Comunidad Autónoma de Galicia $(N=229)$, lo que representa un $61,14 \%$ de la población total.

\section{Instrumento}

El cuestionario final elaborado quedó formado por un total de 51 ítems distribuidos en cuatro bloques de información (Anexo):
- Primer Bloque: Marco contextual de referencia. Identificación del centro educativo (7 ítems). Datos personales, académicos y profesionales (12 ítems). Formato de respuesta cerrada.

- Segundo Bloque: Marco teórico-conceptual. Pensamiento implícito de los orientadores en la atención a la diversidad (12 ítems). Formato de respuesta cerrada.

- Tercer Bloque: Marco práctico. La diversidad en los centros (10 ítems). Formato de respuesta cerrada. 
Alonso, J. D., Castedo, A. L. \& Juste, M. P. (2013). Diseño y Propiedades Psicométricas de un Cuestionario de Opinión sobre la Atención a la Diversidad (CUADIVER).

- Cuarto Bloque: Marco valorativo. Valoración de las medidas de atención a la diversidad (9 ítems). Formato de respuesta escala tipo Likert.

\section{Procedimiento}

El proceso de elaboración del cuestionario se inició con muchas dificultades por su amplitud y complejidad que afectan fundamentalmente a su validez y fiabilidad (Popham, 2002), siendo conscientes en todo momento de los pasos a realizar: (a) análisis de las variables de entrada, proceso y contexto en los propios centros educativos; (b) diseño del cuestionario de opinión; y (c) validación del instrumento. Se intenta en todo momento que el cuestionario resulte lo más completo posible y sin lagunas, tratando de evitar: la exhaustividad excesiva en la lista de ítems, la repetición o colocación inadecuada de los mismos en los distintos ámbitos, la omisión de ítems importantes y la utilización de una terminología difícil e imprecisa en la formulación del mismo.

La primera parte, encaminada a la recogida de información básica de manera participativa sobre aspectos concretos para conocer el estado de la cuestión a estudio y sentar las bases para la posterior construcción del cuestionario, se lleva a cabo con una técnica que trata de captar la realidad social a partir del debate o la discusión en pequeños grupos reproduciendo aquello que sucede en la sociedad (macrosituación) - en nuestro caso los centros educativos -, a través de un grupo de personas (microsituación) reunidas a propósito por el investigador para hablar de un tema concreto (Rubio \& Varas, 1999). En su desarrollo se siguieron las fases señaladas por Gaitán y Piñuel (1998) para los grupos de discusión: previa (planificación de la actividad), intermedia o procesual (realización de la actividad) y final (logro de los objetivos).

Posteriormente, dado que la información obtenida fue abundante y diversa, se procede a sintetizarla y estructurarla a través del programa informático Aquad 5.0, lo cual permitirá un mejor manejo y mayor objetividad al seleccionar las frecuencias de contenido de la misma (Huber \& Marcelo, 1991). Así pues, al unir la información obtenida a la revisión exhaustiva y completa de la literatura pertinente e indagaciones exploratorias preliminares realizadas, se realiza una primera aproximación al cuestionario.

Este protocolo inicial se envía a un grupo de expertos para que lo examinen y transmitan sus consejos, sugerencias y opiniones respecto a la estructura, preguntas, $\mathrm{u}$ otros aspectos que puedan mejorarlo. Se somete pues, a una segunda técnica cualitativa de previsión grupal (método Delphi) que se nutre del juicio de expertos (Landeta, 1999). En nuestro caso, el grupo de expertos está formado por tres doctores de la universidad entendidos en la temática objeto de estudio - educación especial, diversidad, interculturalidad, orientación -, un doctor especialista en procesos metodológicos, un inspector de educación, tres jefes de departamento de orientación, un director de centro, cuatro tutores con diversidad en sus aulas y tres profesores no tutores, con conocimientos, características y experiencia en este campo, a los cuales se solicita y agradece su colaboración. Se les proporciona el protocolo inicial elaborado para que valoren su estructura, contenido y forma, con la posibilidad de incluir o suprimir los aspectos que consideren oportunos.

Una vez realizadas las aclaraciones pertinentes, se pasa por sus despachos o centros a recoger y comentar las aportaciones realizadas. Con las mismas se procede a estructurar el cuestionario enriqueciéndolo con las incorporaciones, variaciones y sugestiones que se consideraron más relevantes (por su repetición, importancia, legalidad, ...). También se lleva a cabo, antes del formato definitivo, la realización de una prueba o estudio piloto (veinticinco orientadores) a los cuales se les envía el cuestionario para que lo respondan y aporten sus valoraciones en cuanto a la dificultad, complejidad, diseño y motivación del mismo, que nos permite realizar las modificaciones y reajustes definitivos.

Finalmente, dado el creciente acceso a las nuevas tecnologías, dentro y fuera de los centros educativos, el clima favorable para su utilización, y su fácil accesibilidad (Abiodun, 2008), se decide redactar el cuestionario en formato Neobook, y enviarlo por correo electrónico a todos los centros educativos de educación primaria que contaban con departamento de orientación.

\section{Análisis Estadísticos}

- En una primera etapa se recolectan y detallan los datos cualitativos:

- Revisión exhaustiva y completa de la literatura y bases de datos.

- Grupos focales en los centros educativos.

- Programa informático Aquad 5.0 para observar las frecuencias de contenido en el discurso y categorizar los datos.

- En la segunda etapa se recaban y analizan los datos cuantitativos:

- Análisis descriptivos y exploratorios de los datos para examinar las distribuciones (medias y porcentajes)

- Aproximación analítica inferencial para ver en que medida los resultados obtenidos pueden ser extrapolados a la población de referencia (prueba $t$, ANOVA, Scheffé).

- Análisis factorial: Primeramente se observa si la matriz puede ser factorizada mediante la medida de adecuación muestral de Kaiser-Meyer-Olkin (KMO) y la prueba de esfericidad de Bartlett. A continuación, se analiza la varianza total y la rotación factorial a través del análisis de los componentes principales y del método de rotación Normalización Varimax con Kaiser. 


\section{Resultados}

Del contenido emergente de los grupos focales sobre la realidad de los centros educativos, una vez filtrado a través del programa Aquad (Tabla 1), se observa que la frecuencia obtenida en los aspectos desfavorables sobre la atención a la diversidad $(63,8 \%)$ es superior a la alcanzada en los aspectos favorables (36,2\%). También se pone de relieve que las aportaciones al campo conceptual (17\%) son menores que las del campo práctico $(83 \%)$.

Tabla 1

Frecuencia de Códigos (Aquad 5.0)

\begin{tabular}{lccc}
\hline & Aspectos desfavorables & Negativos - Mediocres & Total \\
\cline { 2 - 3 } Análisis de la realidad & Aspectos favorables & 19 & 25 \\
\cline { 3 - 3 } & & Prometedores - Positivos & 25 \\
\hline Marco conceptual & & 14 & 10 \\
Marco práctico & 50 \\
\hline
\end{tabular}

Nota. Elaboración propia.

Entre los aspectos favorables cabe señalar que los orientadores conceden gran importancia y muestran preocupación hacia la atención del alumnado con necesidades educativas específicas, destacando la necesidad de su inclusión dentro del grupo clase, además de considerar positiva la incorporación de las nuevas tecnologías de la información y la comunicación en el proceso de enseñanza-aprendizaje de estos alumnos. Sin embargo, entre los desfavorables destacan la escasa formación del profesorado, el tiempo y la actitud del profesorado ante esta temática, la falta de trabajo colaborativo y la tendencia de los profesores a preferir los grupos homogéneos, así como la falta de recursos humanos y materiales. También denuncian el rol pasivo de la administración en el proceso de la atención a la diversidad y el diseño de un sistema educativo que incluya a todo el alumnado sin excepciones.

Con objeto de medir la fiabilidad del cuestionario, se ha calculado el Coeficiente Alfa de Cronbach por ser un indicador de su consistencia interna (Muñiz, 1992). Así, teniendo en cuenta la amplitud del cuestionario, el índice obtenido en la Alfa de Cronbach es muy bueno (.92), similar al obtenido en estudios similares más recientes (Biencinto-López, González-Barbera, García-García, Sánchez-Delgado, \& Madrid-Vivar, 2009). También se utilizó el método de las dos mitades obteniendo una correlación en los ítems pares de .91 (muy aceptable), y en los impares .82 (aceptable).

No obstante, siendo la fiabilidad una característica necesaria e imprescindible de los instrumentos de medida, no es suficiente para defender la legitimidad de los datos recogidos. Es necesario también que el instrumento sea válido (Abell, Springer, \& Kamata, 2009), es decir, que se adecue a los objetivos principales de la investigación
(Cea D'Ancona, 1999). Para ello fue elaborado bajo una cuidadosa planificación y objetividad con la triangulación de múltiples indicadores (obtenidos a través de revisiones bibliográficas, grupos focales, consultas a expertos y pretest) que cubren el campo de la diversidad - validez de contenido.

Por último, se estudia la validez de constructo del cuestionario, realizando su análisis factorial por el método de componentes principales (Rotación Varimax con Kaiser). Previamente, en cada bloque de contenido (teóricoconceptual, práctico, y valorativo) se calcula el índice de Kaiser-Meyer-Olkin (KMO) y la prueba de Bartlett para confirmar si la muestra es aceptable y puede ser factorizada. Además, en el análisis factorial de los ítems realizado para la selección de variables de cada factor, se recogen solamente las saturaciones superiores a .40 .

En el marco teórico-conceptual, el índice $\mathrm{KMO}=.703$ y la prueba de Bartlett $\left(\chi^{2}=78.794, p=.000\right)$ confirman que la muestra puede ser factorizada. Del análisis realizado se extraen un total de cuatro factores que explican un $49,9 \%$ de la varianza de los datos (Tabla 2).

El primer factor $\left(F_{1}=14,2 \%\right.$ de la varianza), define la concepción general de la diversidad. Por su parte, el segundo factor $\left(F_{2}=12,9 \%\right.$ de la varianza) se relaciona con las variables normativo-legislativas en este campo. El tercer factor $\left(F_{3}=12,1 \%\right.$ de la varianza $)$ integra las variables que hacen referencia a los indicadores de la atención a la diversidad. Finalmente, el cuarto factor $\left(F_{4}=10,7 \%\right.$ de la varianza), recoge las variables relacionadas con la predisposición que existe en los centros hacia la atención del alumnado diverso.

El marco práctico se ha desglosado en cuatro grupos (colectivos, actividades, cambios/mejoras y recursos), realizando un análisis factorial en cada uno de ellos (Tabla 3). 
Alonso, J. D., Castedo, A. L. \& Juste, M. P. (2013). Diseño y Propiedades Psicométricas de un Cuestionario de Opinión sobre la Atención a la Diversidad (CUADIVER).

Tabla 2

Estructura Factorial (método ejes principales, rotación varimax) del Marco Teórico-Conceptual

\begin{tabular}{|c|c|c|c|c|}
\hline Ítems & $\mathrm{F} 1$ & $\mathrm{~F} 2$ & F3 & F4 \\
\hline Concepción & .734 & & & \\
\hline Actualidad & .561 & & & \\
\hline Práctica & .538 & & & \\
\hline Formación & & .780 & & \\
\hline Legislación & & .669 & & \\
\hline Ley Orgánica de Calidad & & .505 & & \\
\hline Diversidad & & & .782 & \\
\hline Calidad & & & .624 & \\
\hline Finalidad & & & .567 & \\
\hline Multiculturalidad & & & & .687 \\
\hline Actitud & & & & .571 \\
\hline Principios & & & & .531 \\
\hline Varianza & 1.705 & 1.543 & 1.457 & 1.286 \\
\hline$\%$ Var. & 14.221 & 12.856 & 12.141 & 10.720 \\
\hline
\end{tabular}

Nota. Elaboración propia.

Tabla 3

Estructura Factorial (método ejes principales, rotación varimax) del Marco Práctico

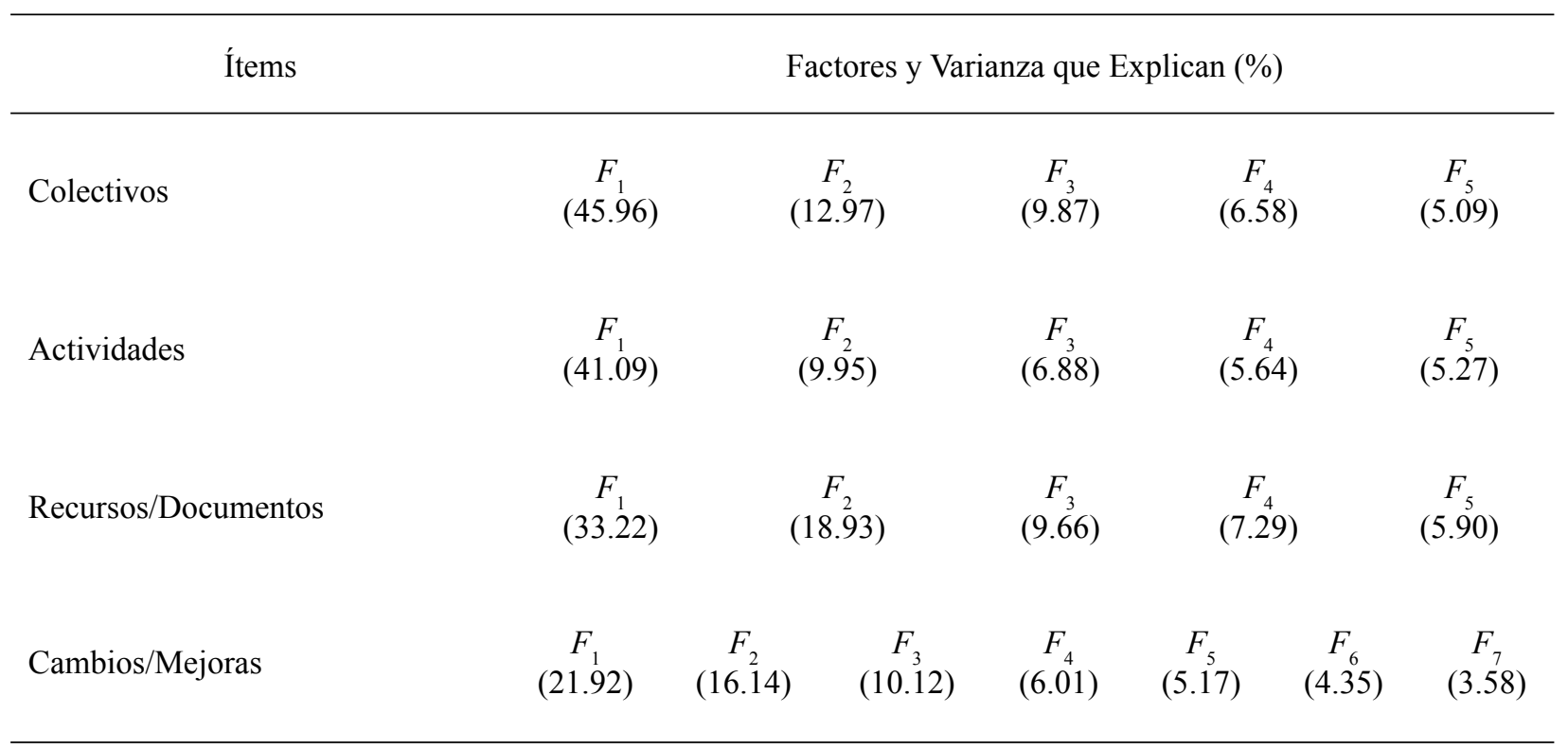

Nota. Elaboración propia. 
En el grupo de colectivos, las medidas previas (KMO $\left.=.75 ; \chi^{2}=1023.55 ; p=.000\right)$ confirman la aceptación de la muestra y factorización de la matriz. El análisis factorial indica la existencia de cinco factores que explican un $80,3 \%$ de la varianza de los datos. Los distintos factores obtenidos aparecen ordenados por el mayor grado de colaboración de los colectivos con el departamento de orientación en la atención de la diversidad: profesores tutores, profesores de apoyo, especialistas en pedagogía terapéutica (PT) o audición y lenguaje (AL), equipo directivo, inspección, administración, familias, y alumnado.

En el grupo de actividades, confirmada la aceptación y factorización $\left(\mathrm{KMO}=.85 ; \chi^{2}=1046.14 ; p=.000\right)$, se pone de manifiesto la existencia de cinco factores que explican el $68.8 \%$ de la varianza total. Estos factores se corresponden con las siguientes dimensiones: Informes y diagnósticos $\left(F_{1}=41.1 \%\right)$, colaboración y asesoramiento $\left(F_{2}=9.9 \%\right)$, material $\left(F_{3}=6.9 \%\right)$, información $\left(F_{4}=5.6 \%\right)$ y actuaciones $\left(F_{5}=5.3 \%\right)$. Se observa aquí, un fuerte predominio de actuaciones propias de un modelo clínico y una menor colaboración en los programas a familias y asesoramiento en la organización de las aulas (modelo preventivo).

En el apartado de recursos y documentos existentes en los centros $\left(\mathrm{KMO}=.77 ; \chi^{2}=1057.46 ; p=.000\right)$, se obtuvieron cinco factores que vienen a explicar el $75 \%$ de la varianza total, correspondiendo a las dimensiones: metodología $\left(F_{1}=33.2 \%\right)$, material $\left(F_{2}=18.9 \%\right)$, documentos del centro $\left(F_{3}=9.7 \%\right)$, espacios $\left(F_{4}=7.3 \%\right)$ y colectivos
$\left(F_{5}=5.9 \%\right)$. Reseñar que la administración dota a los centros en función del tipo y grado de necesidad específica que presente el alumnado de referencia.

En el último grupo, cambios y mejoras $(\mathrm{KMO}=.75$; $\left.\chi^{2}=1769.99 ; p=.000\right)$, aparecen siete factores que explican $67.3 \%$ de la varianza, correspondiéndose con las dimensiones: organización $\left(F_{1}=21.9 \%\right)$, currículum $\left(F_{2}=16.1 \%\right)$, obstáculos $\left(F_{3}=10.1 \%\right)$, agrupamientos $\left(F_{4}=6.0 \%\right)$, estructura $\left(F_{5}=5.2 \%\right)$, planificación $\left(F_{6}=4.4 \%\right)$ y recursos $\left(F_{7}=3.6 \%\right)$.

Señalar que dentro de los cambios introducidos en los centros para la atención a la diversidad, lo que más se ha realizado fue la integración (sobre todo física) del alumnado en su grupo-clase. En referencia a las mejoras a introducir, se centran en la formación del profesorado y en la acción tutorial, siendo los principales obstáculos el trabajo en equipo y la falta de motivación del profesorado.

Finalmente, el marco valorativo arrojó una medida de adecuación muestral $\mathrm{KMO}=.88$, con la prueba de Bartlett estadísticamente significativa $\left(\chi^{2}=397.07 ; p=.000\right)$. Aquí, el análisis de componentes principales nos muestra una escala bifactorial con autovalores que excedieron de la unidad y arrojan una variación total de $71,4 \%$. Se observa que la mayor varianza extraída recae sobre el primer factor con un autovalor de 5.39 siendo considerado el factor principal. El segundo factor que emerge en este análisis presenta un autovalor de 1.03 (Tabla 4).

Tabla 4

Estructura Factorial (método ejes principales, rotación varimax) del Marco Valorativo

\begin{tabular}{lcc}
\hline \multicolumn{1}{c}{ Ítems } & Factor 1 & Factor 2 \\
\hline Acción tutorial y orientadora & .879 & \\
Medidas ordinarias a nivel de aula & .873 & \\
Refuerzo Educativo o Adaptaciones curriculares no significativas & .785 & \\
Medidas ordinarias de atención a las minorías étnicas & .770 & \\
Medidas organizativas & .741 & \\
Medidas ordinarias a nivel de centro & .711 & \\
Adaptaciones curriculares significativas (ACI) & & .810 \\
Medidas extraordinarias de atención a la diversidad cultural & & .793 \\
Permanencia o reducción de un año más en el ciclo & & .678 \\
Varianza & & 1.03 \\
\% Varianza & 5.39 & 11.4 \\
\hline
\end{tabular}

Nota. Elaboración propia.

Los resultados obtenidos muestran un primer factor $\left(F_{1}=.88 / .71\right)$ que agrupa los ítems referentes a las medidas curriculares de carácter ordinario, mientras que el segundo factor $\left(F_{2}=.81 / .68\right)$ agrupa las medidas curriculares extraordinarias que se identifican con las actuaciones extremas en la atención del alumnado diverso, consideradas de difícil aplicación y que marcan la competencia del individuo en cuanto a su capacidad de enfrentamiento y preparación adecuada.

\section{Discusión y Conclusiones}

Una de las principales metas del sistema educativo actual, es la apuesta por la máxima atención al alumnado 
Alonso, J. D., Castedo, A. L. \& Juste, M. P. (2013). Diseño y Propiedades Psicométricas de un Cuestionario de Opinión sobre la Atención a la Diversidad (CUADIVER).

diverso existente en los centros educativos desde un marco de igualdad de oportunidades, sin embargo en su práctica suele provocar dudas, efectos contrarios, frustración escolar e incluso abandono y exclusión social. Por consiguiente es urgente prestar un apoyo sistemático y real a las necesidades cognitivas, afectivas y sociales de los colectivos más desfavorecidos en los centros de enseñanza. La falta de instrumentos rigurosos en el mercado para obtener información sobre cómo se están desarrollando en los centros educativos las medidas de atención a la diversidad, nos lleva a diseñar un cuestionario (CUADIVER) que sea capaz de diagnosticar y valorar los distintos enfoques educativos en la atención del alumnado con dificultades de aprendizaje, permitiendo a su vez, ayudar a mejorar sus propuestas didácticas, curriculares y organizativas. El reto pues, es doble, por un lado divulgar y sensibilizar la atención del alumnado con necesidades educativas específicas $\mathrm{y}$, por otro, proporcionar a las comunidades escolares un recurso más que sirva de ayuda para comprobar las actuaciones en este campo.

El cuestionario en su conjunto presenta una solidez excelente, unas buenas propiedades de comprensibilidad, aceptación y consistencia (Flick, 2004; Sandín, 2000), con un tiempo promedio invertido en la cumplimentación de veinte minutos. El modo ideal de utilización es el autocumplimentado que lo convierte en un instrumento de fácil uso en la práctica educativa. Su validez de constructo, medida por los pesos factoriales de los ítems (entre .50 y .88) puede ser considerada como buena, representando los factores extraídos adecuadamente a las dimensiones teóricas.

Por otro lado, la estructura factorial del CUADIVER se revela muy estable. Así pues, los factores que componen el instrumento representan los aspectos más relevantes y diferenciados de las percepciones relacionadas con la atención a la diversidad (Pino, Domínguez, \& LópezCastedo, 2007). El primer paso, realizado desde dentro y en la escuela - investigación educativa -, muestra una realidad desfavorable en esta parcela con escasa formación del profesorado, falta de trabajo colaborativo y recursos (humanos y materiales), y una ligera tendencia hacia la preferencia de los grupos homogéneos. También delata el rol pasivo que está asumiendo la administración y el diseño de un sistema educativo más selectivo que comprensivo.

El segundo paso, realizado desde fuera y para la escuela - investigación en educación - (Cabello, 1999), nos ha permitido conocer "las actitudes, las creencias, los valores, las características demográficas, los comportamientos, las opiniones, los hábitos, los deseos y las ideas de las personas; además de conseguir otro tipo de información" (McMillan \& Schumacher, 2005, p. 292). Aquí, los resultados obtenidos permiten identificar dos tipos de centros a la hora de enfrentarse a la creciente diversidad de su alumnado: en un primer grupo (mayoritario), los que identifican las diferencias y diseñan programas ajustados para un grupo concreto de estudiantes (prácticas educa- tivas integradoras); y en un segundo grupo (minoritario), los que impulsan una educación de calidad con equidad y excelencia para todo el alumnado (prácticas educativas inclusoras).

Por último reseñar que el cuestionario CUADIVER se presenta, dentro del vacío de instrumentos que existe en este campo, como un instrumento imprescindible para su utilización en la evaluación e intervención para la mejora de la atención a la diversidad en los centros de enseñanza (considerada hoy en día una de las prioridades del sistema educativo) y es, además, una herramienta que aporta a esta temática mayor coherencia teórica, metodológica y práctica, con valores (de fiabilidad y validez) muy próximos a los obtenidos en otras pruebas similares (Álvarez et al., 2002; Oliver, 1999). En definitiva, este cuestionario pone de manifiesto que es un instrumento con unas prestaciones psicométricas muy satisfactorias y útil para la supervisión del estado de la atención a la diversidad en cualquier centro de enseñanza.

Es cierto que nuestra investigación tiene limitaciones, una de las cuales es la que deriva de la muestra, que no es representativa de los orientadores españoles en la educación primaria, sino sólo de la Comunidad Autónoma Gallega Sería, pues, deseable ampliar la validación del cuestionario a una muestra representativa del sistema educativo no universitario español, lo que es una tarea sumamente compleja En este sentido, la necesidad de avanzar en la transformación de los centros educativos en verdaderas comunidades inclusivas se convierte en el estanque sobre el cual deben beber las actuales políticas y prácticas educativas.

\section{Referencias}

Abell, N., Springer, D. W., \& Kamata, A. (2009). Developing and validating rapid assessment instruments. Oxford, UK: Oxford University Press.

Abiodun, B. (2008). Análisis de fiabilidad y de factores de una Escala de Eficacia Docente para Profesores de la Enseñanza Secundaria de Nigeria. Revista Electrónica de investigación Psicoeducativa, 16, 6(3), 823-846.

Álvarez, V., Rodríguez, A., García, E., Gil, J., López, I., Romero, S., ...Correa, J. (2002). La atención a la diversidad en los centros de enseñanza secundaria: estudio descriptivo en la provincia de Sevilla. Revista de Investigación Educativa, 20(1), 225-245.

Axinn, W. G., \& Pearce, L. D. (2006). Mixed method data collection strategies. Cambridge, MA: Cambridge University Press.

Barbour, R. (2007). Doing focus groups. London: Sage.

Biencinto-López, C., González-Barberá, C., García-García, M., Sánchez-Delgado, P., \& Madrid-Vivar, D. (2009). Diseño y propiedades psicométricas del AVACO-EVADIE. Cuestionario para la evaluación de la atención a la atención a la diversidad como dimensión educativa en las instituciones escolares. Relieve, 15(1), 1-36.

Bisquerra, R. (2000). Métodos de investigación educativa. Guía práctica. Barcelona, España: Centro de Estudios a Crédito.

Boeije, H. (2009). Analysis in qualitative research. London, UK: Sage. 
Cabello, M. J. (1999). Prácticas, teorías e investigaciones: descubriendo lo que es posible. In E. Rubio Rivera \& L. Rayón Rumayor (Eds.), Repensar la enseñanza desde la diversidad: Vol. 9. Cuadernos de Cooperación Educativa (pp. 263-266). Sevilla, España: Movimiento Cooperativo de Educación Popular.

Casanova, M. A. (1999). Educación para una sociedad plural. Organización y Gestión Educativa, 2, 3-7.

Cea D'Ancona, M. A. (1999). Metodología cuantitativa. Estrategias y técnicas de investigación social. Madrid, España: Síntesis.

Collins, K. M. T., Onwuegbuzie, A. J., \& Sutton, I. L. (2006). A model incorporating the rationale and purpose for conducting mixed methods research in special education and beyond. Learning Disabilities: A Contemporary Journal, 4, 67-100.

Creswell, J. W. (2009). Research design: Qualitative, quantitative and mixed approaches. Thousand Oaks, CA: Sage.

Domínguez, J. (2009). Atención a la diversidad en la educación primaria: evolución y situación actual. Barcelona, España: Editorial Davinci.

Essomba, M. A. (2003). Els discursos sobre atenció a la diversitat en la comunitat educativa a Catalunya (Tesis doctoral, Universidad Autónoma de Barcelona, España).

Flick, U. (2004). Introducción a la investigación cualitativa. Madrid, España: Morata.

Gaitán, J. A., \& Piñuel, J. L. (1998). Técnicas de investigación en comunicación social. Elaboración y registro de datos. Madrid, España: Síntesis.

Hernández, R., \& Mendoza, C. P. (2008). El matrimonio cuantitativo-cualitativo: el paradigma mixto. Documento presentado en el Sexto Congreso de Investigación en Sexología, Villahermosa, Tabasco, México.

Huber, G., \& Marcelo, C. (1991). Computer assistance for testing hypotheses about qualitative data: The software package AQUAD 3.0. Qualitative Sociology, 14(4), 325-347.

Jiménez, C., \& González, M. A. (2010). Pedagogía Diferencial $y$ atención a la diversidad. Madrid, España: Universidade Nacional de Educação a Distância.

Justicia, F., Benítez, J. L., Pichardo, M. C., Fernández, E., García, T., \& Fernández, M. (2006). Aproximación a un nuevo modelo explicativo del comportamiento antisocial. Revista Electrónica de Investigación Psicoeducativa, 9, 4(2), 131-150.

Land, T. (2005). The qualitative-quantitative distintion: Some comments. Scandinavian Journal of Educational Research, 49(2), 115-132.

Landeta, J. (1999). El método Delphi. Una técnica de previsión para la incertidumbre. Barcelona, España: Ariel.

Marchena, C. (2002). Náufragos educativos. Escuela Española, $3.556(1466), 2$.

McMillan, J. H., \& Schumacher, S. (2005). Investigación educativa. Una introducción conceptual (5. ed.). Madrid, España: Pearson Educación.

Muñiz, J. (1992). Teoría clásica de los tests. Madrid, España: Pirámide.

Nicolaci da Costa, A. M. (2007). O campo da pesquisa qualitativa e o método de explicitação do Discurso Subjacente (MEDS). Psicologia: Reflexao e Critica, 20(1), 65-73.

Oliver, M. C. (1999). La atención a la diversidad desde los agrupamientos flexibles de alumnos (Tesis doctoral, Universidad de Barcelona, España).
Pino, M., Domínguez, J., \& López-Castedo, A. (2007). Evaluating appreciation of measures attending to pupil diversity (EMAD). Psychological Reports, 100, 783-786.

Popham, W. J. (2002). Classroom assessment, what teachers need to know. Boston, MA: Allyn \& Bacon.

Rubio, M. J., \& Varas, J. (1999). El análisis de la realidad en la intervención social. Métodos y técnicas de investigación (2. ed.). Madrid, España: Editorial Central Catequística Salesiana.

Sandín, M. P. (2000). Criterios de validez en la investigación cualitativa: de la objetividad a la solidaridad. Revista de Investigación Educativa, 18(1), 223-242.

Slonim-Nevo, V., \& Nevo, I. (2009). Conflicting findings in mixed methods research: An illustration from an Israeli study on immigration. Journal of Mixed Methods Research, 3(2), 109-128.

Stake, R. E. (1998). Investigación con estudio de casos. Madrid, España: Morata. 
Alonso, J. D., Castedo, A. L. \& Juste, M. P. (2013). Diseño y Propiedades Psicométricas de un Cuestionario de Opinión sobre la Atención a la Diversidad (CUADIVER).

\section{ANEXO}

\section{CUADIVER [Cuestionario de la atención a la diversidad]}

PRIMER BLOQUE: MARCO CONTEXTUAL

\section{IDENTIFICACIÓN DEL CENTRO}

\begin{tabular}{|c|c|c|}
\hline \multicolumn{3}{|c|}{ 1. TIPO DE CENTRO } \\
\hline & Público & $\square$ \\
\hline & Concertado & $\square$ \\
\hline & Privado & $\square$ \\
\hline \multicolumn{3}{|c|}{ 2. NIVELES IMPARTIDOS EN EL CENTRO } \\
\hline & Educación Infantil y Primaria (CEIP) & $\square$ \\
\hline & Educación Primaria & $\square$ \\
\hline & Educación infantil, primaria y secundaria (CPI) & $\square$ \\
\hline & Educación Secundaria & $\square$ \\
\hline \multicolumn{3}{|c|}{ 3. PROVINCIA } \\
\hline & La Coruña & $\square$ \\
\hline & Lugo & $\square$ \\
\hline & Orense & $\square$ \\
\hline & Pontevedra & $\square$ \\
\hline \multicolumn{3}{|c|}{ 4. PROCEDENCIA SOCIOGEOGRÁFICA DEL ALUMNADO } \\
\hline & Rural (menos de 10.000 habitantes) & $\square$ \\
\hline & Semiurbana (entre 10.000 y 50.000 habitantes) & $\square$ \\
\hline & Urbana (más de 50.000 habitantes) & $\square$ \\
\hline \multicolumn{3}{|c|}{ 5. NÚMERO DE UNIDADES DEL CENTRO } \\
\hline & Centros de 40 o más unidades & $\square$ \\
\hline & Centros de 18 a 39 unidades & $\square$ \\
\hline & Centros de 12 a 17 unidades & $\square$ \\
\hline & Centros de 6 a 11 unidades & $\square$ \\
\hline & Centros de 3 a 5 unidades & $\square$ \\
\hline \multicolumn{3}{|c|}{ 6. NÚMERO DE ALUMNOS/AS } \\
\hline & Menos de 70 & $\square$ \\
\hline & De 70 a 150 & $\square$ \\
\hline & De 151 a 200 & $\square$ \\
\hline & De 201 a 300 & $\square$ \\
\hline & Más de 300 & $\square$ \\
\hline
\end{tabular}




\section{DATOS PERSONALES, ACADÉMICOS Y PROFESIONALES}

\begin{tabular}{|c|c|c|}
\hline \multicolumn{3}{|l|}{ 7. SEXO } \\
\hline & Hombre & $\square$ \\
\hline & Mujer & $\square$ \\
\hline \multicolumn{3}{|l|}{ 8. EDAD } \\
\hline & Menos de 30 años & $\square$ \\
\hline & De 30 a 40 años & $\square$ \\
\hline & De 41 a 50 años & $\square$ \\
\hline & De 51 a 60 años & $\square$ \\
\hline & Más de 60 años & $\square$ \\
\hline \multicolumn{3}{|c|}{ 9. AÑOS DE EXPERIENCIA DOCENTE } \\
\hline & Ninguno & $\square$ \\
\hline & Menos de 3 años & $\square$ \\
\hline & De 3 a 5 años & $\square$ \\
\hline & De 6 a 10 años & $\square$ \\
\hline & De 11 a 15 años & $\square$ \\
\hline & De 16 a 20 años & $\square$ \\
\hline & Más de 20 años & $\square$ \\
\hline \multicolumn{3}{|c|}{ 10. AÑOS DE EXPERIENCIA EN ORIENTACIÓN } \\
\hline & Menos de 3 años & $\square$ \\
\hline & De 3 a 5 años & $\square$ \\
\hline & De 6 a 10 años & $\square$ \\
\hline & Más de 10 años & $\square$ \\
\hline \multicolumn{3}{|c|}{ 11. TITULACIONES ACADÉMICAS (Múltiple) } \\
\hline & Diplomatura en Magisterio & $\square$ \\
\hline & Licenciatura en Pedagogía & $\square$ \\
\hline & Licenciatura en Psicopedagogía & $\square$ \\
\hline & Licenciatura en Psicología & $\square$ \\
\hline \multicolumn{3}{|c|}{ 12. SISTEMA DE ACCESO AL CARGO DE JEFE/A DE DEPARTAMENTO DE ORIENTACIÓN } \\
\hline & Concurso específico para miembros de los antiguos equipos & $\square$ \\
\hline & Derecho preferente al centro (Licenciatura) & $\square$ \\
\hline & $\begin{array}{l}\text { Derecho preferente al centro (Antiguas escuelas universitarias de } \\
\text { psicología hasta 1974) }\end{array}$ & $\square$ \\
\hline & Derecho preferente a la zona educativa & $\square$ \\
\hline & Concurso general de traslados para jefaturas de departamento & $\square$ \\
\hline & Concurso/Oposición libre o restringida para maestros & $\square$ \\
\hline
\end{tabular}


Alonso, J. D., Castedo, A. L. \& Juste, M. P. (2013). Diseño y Propiedades Psicométricas de un Cuestionario de Opinión sobre la Atención a la Diversidad (CUADIVER).

\begin{tabular}{|l|r|r|}
\hline 13. MIEMBROS QUE FORMAN PARTE DEL DEPARTAMENTO DE ORIENTACIÓN (Múltiple) \\
\hline & Tutores & $\square$ \\
\hline & Especialistas en pedagogía terapeutica & $\square$ \\
\hline & Especialistas en audición y lenguaje & $\square$ \\
\hline & Profesor de formación y orientación laboral & $\square$ \\
\hline & Jefes de departamentos de orientación de colegios adscritos & $\square$ \\
\hline & Coordinadores de ciclo & $\square$ \\
\hline & Responsable o director de centros incompletos & $\square$ \\
\hline 14. NÚMERO DE CENTROS ADSCRITOS AL DEPARTAMENTO DE ORIENTACIÓN \\
\hline \\
\hline
\end{tabular}

\section{SEGUNDO BLOQUE: MARCO CONCEPTUAL, PRÁCTICO Y VALORATIVO}

\section{PENSAMIENTO IMPLÍCITO SOBRE LA ATENCIÓN A LA DIVERSIDAD}

\begin{tabular}{|l|l|c|}
\hline \multirow{2}{*}{$\begin{array}{l}\text { 15. DE LOS SIGUIENTES } \\
\text { INDICADORES }\end{array}$} & Contar con un marco de actuación regulamentado y flexible & $\square$ \\
\cline { 2 - 3 } $\begin{array}{l}\text { CUÁL ES PARA } \\
\text { TI EL MEJOR }\end{array}$ & Bajar la proporción profesorado/alumnado & $\square$ \\
\hline & Dar respuesta adecuada a la diversidad & $\square$ \\
\hline & Incentivar las tareas del profesorado & $\square$ \\
\hline $\begin{array}{l}\text { 16. ATENDER A LA } \\
\text { DIVERSIDAD } \\
\text { ES ... }\end{array}$ & $\begin{array}{l}\text { Adecuar el currículo al momento evolutivo del niño/a poniéndose a su } \\
\text { nivel de conocimientos y hacerlo avanzar }\end{array}$ & $\square$ \\
\cline { 2 - 3 } & $\begin{array}{l}\text { Hacer una adaptación de los objetivos, contenidos, metodología, } \\
\text { actividades, del curso según las necesidades educativas de cada alumno/a }\end{array}$ & $\square$ \\
\hline & $\begin{array}{l}\text { Conjunto de medidas educativas planificadas para procurarle al alumnado } \\
\text { máxima socialización e individualización }\end{array}$ & $\square$ \\
\hline \multirow{2}{*}{$\begin{array}{l}\text { 17. LA CONCEPCIÓN } \\
\text { GENERAL QUE TIENES } \\
\text { SOBRE LA DIVERSIDAD ES } \\
\text { PREDOMINANTEMENTE... }\end{array}$} & Organizativa & $\square$ \\
\hline & Metodológica & $\square$ \\
\hline & Curricular & $\square$ \\
\hline
\end{tabular}




\begin{tabular}{|c|c|c|}
\hline \multirow{4}{*}{$\begin{array}{l}\text { 18. EL FIN DE LA } \\
\text { ATENCIÓN } \\
\text { A LA DIVERSIDAD } \\
\text { ES ... }\end{array}$} & Potenciar la igualdad de oportunidades & $\square$ \\
\hline & La aceptación de la comprensibilidad & $\square$ \\
\hline & Fomentar la heterogeneidad del grupo frente a la homogeneidad & $\square$ \\
\hline & Ayudar al alumnado con dificultades de aprendizaje & $\square$ \\
\hline \multirow{5}{*}{$\begin{array}{l}\text { 19. EN LA ACTUALIDAD } \\
\text { LAS MEDIDAS DE } \\
\text { ATENCIÓN AL ALUMNADO } \\
\text { DIVERSO ... }\end{array}$} & Son buenas pero difíciles de llevar a la práctica & $\square$ \\
\hline & Son eficaces siempre que tengamos claros los objetivos & $\square$ \\
\hline & Son positivas si el profesorado las conoce bien & $\square$ \\
\hline & $\begin{array}{l}\text { Necesitan mucha preparación y coordinación y, por tanto tiempo, } \\
\text { para ser eficaces }\end{array}$ & $\square$ \\
\hline & Son negativas, al provocar efectos no deseados en el alumnado & $\square$ \\
\hline \multirow{4}{*}{$\begin{array}{l}\text { 20. EL MARCO } \\
\text { LEGISLATIVO QUE SE } \\
\text { ENCARGA DE LEGITIMAR } \\
\text { LA DIVERSIDAD ... }\end{array}$} & Es aceptable para poder llevarla a la práctica & $\square$ \\
\hline & Está bien en el aspecto teórico, pero en la práctica fracasa & $\square$ \\
\hline & Está descontextualizado, falto de realismo y de previsión & $\square$ \\
\hline & Es complejo, y resulta inviable su aplicación & $\square$ \\
\hline \multirow{4}{*}{$\begin{array}{l}\text { 21. LAS ACTIVIDADES } \\
\text { DE FORMACIÓN EN EL } \\
\text { ÁMBITO DE LA } \\
\text { DIVERSIDAD RESPONDEN } \\
\text { A TUS NECESIDADES }\end{array}$} & Nada & $\square$ \\
\hline & Poco & $\square$ \\
\hline & Bastante & $\square$ \\
\hline & Mucho & $\square$ \\
\hline \multirow{5}{*}{$\begin{array}{l}\text { 22. CUAL CONSIDERAS } \\
\text { COMO PRINCIPIO CLAVE } \\
\text { EN LA RESPUESTA A LA } \\
\text { DIVERSIDAD }\end{array}$} & La flexibilidad metodológica & $\square$ \\
\hline & La organización de los agrupamientos & $\square$ \\
\hline & La motivación y autoestima & $\square$ \\
\hline & La anticipación y previsión & $\square$ \\
\hline & La adaptación curricular & $\square$ \\
\hline \multirow{4}{*}{$\begin{array}{l}\text { 23. LA MEJOR PRÁCTICA } \\
\text { EDUCATIVA PARA } \\
\text { ATENDER LA DIVERSIDAD } \\
\text { EN EL AULA ES ... }\end{array}$} & Clase magistral, explicación y ejercicios para todos & $\square$ \\
\hline & Clase magistral, explicación y ejercicios con ajustes individuales & $\square$ \\
\hline & Grupos flexibles con ejercicios generales & $\square$ \\
\hline & Estrategia común y actividades graduadas según las dificultades & $\square$ \\
\hline \multirow{4}{*}{$\begin{array}{l}\text { 24. CUÁL ES LA ACTITUD } \\
\text { MÁS NEGATIVA EN EL } \\
\text { TRATAMIENTO DE LA } \\
\text { DIVERSIDAD }\end{array}$} & La indiferencia & $\square$ \\
\hline & La marginación & $\square$ \\
\hline & La asimilación & $\square$ \\
\hline & Otra (decir cual) & $\square$ \\
\hline \multirow{5}{*}{$\begin{array}{l}\text { 25. QUÉ CONSIDERAS } \\
\text { MÁS IMPORTANTE EN LA } \\
\text { MULTICULTURALIDAD ... }\end{array}$} & Tener diseñado un plan de acogida coordinado con la localidad & $\square$ \\
\hline & $\begin{array}{l}\text { Adaptar estilos diferentes de aprendizaje del alumnado } \\
\text { independientemente de su origen étnico o cultural }\end{array}$ & $\square$ \\
\hline & Integrar perspectivas multiculturales en los métodos educativos & $\square$ \\
\hline & $\begin{array}{l}\text { Respetar las diferencias en el modo de aprender del alumnado debido a su } \\
\text { personalidad individual o su grupo cultural }\end{array}$ & $\square$ \\
\hline & Aceptar e incluir las diferencias étnicas y culturales del alumnado & $\square$ \\
\hline
\end{tabular}


Alonso, J. D., Castedo, A. L. \& Juste, M. P. (2013). Diseño y Propiedades Psicométricas de un Cuestionario de Opinión sobre la Atención a la Diversidad (CUADIVER).

\section{LA DIVERSIDAD EN LOS CENTROS EDUCATIVOS}

\begin{tabular}{|c|c|c|c|c|c|c|}
\hline \multicolumn{2}{|c|}{ M (mala), R (regular), N (normal), B (buena), E (excelente) } & \multirow[t]{2}{*}{$\mathbf{M}$} & \multirow[t]{2}{*}{$\mathbf{R}$} & \multirow[t]{2}{*}{$\mathbf{N}$} & \multirow[t]{2}{*}{ B } & $\mathbf{E}$ \\
\hline 26. ACTITUD DE LOS & Equipo directivo & & & & & \\
\hline COLECTIVOS HACIA & Servicio de inspección & & & & & \\
\hline DIVERSIDAD EN EL & Administración & & & & & \\
\hline & Tutores & & & & & \\
\hline & Profesores no tutores & & & & & \\
\hline & Especialistas en PT o AL & & & & & \\
\hline & Profesores de apoyo & & & & & \\
\hline \multirow{7}{*}{$\begin{array}{l}\text { 27. EN QUE } \\
\text { MEDIDA ACUDEN A } \\
\text { SOLICITARLE APOYO O } \\
\text { COLABORACIÓN EN LA } \\
\text { ATENCIÓN } \\
\text { A LA DIVERSIDAD }\end{array}$} & Equipo directivo & & & & & \\
\hline & Tutores & & & & & \\
\hline & Profesorado no tutor & & & & & \\
\hline & Familias & & & & & \\
\hline & Alumnado & & & & & \\
\hline & Especialistas en PT o AL & & & & & \\
\hline & Profesores de apoyo & & & & & \\
\hline \multirow{7}{*}{$\begin{array}{l}\text { 28. CUALES SON } \\
\text { LAS ACTUACIONES } \\
\text { MÁS SOLICITADAS } \\
\text { PARA ATENDER A LA } \\
\text { DIVERSIDAD EN LOS } \\
\text { CENTROS }\end{array}$} & Realizar/confirmar diagnósticos & & & & & \\
\hline & Colaboración en la preparación de RE o ACI & & & & & \\
\hline & Elaborar y preparar material didáctico & & & & & \\
\hline & Asesoramiento en la organización del aula & & & & & \\
\hline & Preparar programas para familias & & & & & \\
\hline & Colaborar en los proyectos de centro $(\mathrm{PEC}, \ldots)$ & & & & & \\
\hline & Realizar informes (Psicopedagógicos) & & & & & \\
\hline \multirow{11}{*}{$\begin{array}{l}\text { 29.EN QUE MEDIDA } \\
\text { ES ADECUADA } \\
\text { LA DOTACIÓN DE } \\
\text { RECURSOS EN } \\
\text { TU CENTRO PARA } \\
\text { LA ATENCIÓN AL } \\
\text { ALUMNADO DIVERSO }\end{array}$} & Aulas adecuadas & & & & & \\
\hline & Libros y revistas especializadas & & & & & \\
\hline & Material de diagnóstico & & & & & \\
\hline & Bibliografía teórico/práctica & & & & & \\
\hline & Mobiliario & & & & & \\
\hline & Cuidadores/as & & & & & \\
\hline & Especialistas en PT y AL & & & & & \\
\hline & Profesores de apoyo & & & & & \\
\hline & Equipo de Orientación Específico & & & & & \\
\hline & Dotaciones económicas & & & & & \\
\hline & Tiempo disponible para la atención a la diversidad & & & & & \\
\hline
\end{tabular}




\begin{tabular}{|c|c|c|c|c|c|c|}
\hline \multicolumn{2}{|c|}{ M (mala), R (regular), N (normal), B (buena), E (excelente) } & \multirow[t]{2}{*}{$\mathbf{M}$} & \multirow[t]{2}{*}{$\mathbf{R}$} & \multirow[t]{2}{*}{$\mathbf{N}$} & \multirow[t]{2}{*}{ B } & \multirow[t]{2}{*}{$\mathbf{E}$} \\
\hline \multirow{9}{*}{$\begin{array}{l}\text { 30. QUE CAMBIOS } \\
\text { SE REALIZARON EN } \\
\text { EL CENTRO PARA } \\
\text { LA ATENCIÓN DE LA } \\
\text { DIVERSIDAD DE SU } \\
\text { ALUMNADO }\end{array}$} & Redistribución de espacios & & & & & \\
\hline & Incremento de los recursos didácticos & & & & & \\
\hline & Cambios en las metodologías & & & & & \\
\hline & Nuevas formas de evaluar los aprendizajes & & & & & \\
\hline & Relación con las familias & & & & & \\
\hline & Relación con instituciones y servicios del entorno & & & & & \\
\hline & Cambios curriculares & & & & & \\
\hline & Cambios organizativos & & & & & \\
\hline & Integración del alumnado en el grupo-clase & & & & & \\
\hline \multirow{13}{*}{$\begin{array}{l}\text { 31. ACTIVIDADES O } \\
\text { TAREAS REALIZADAS } \\
\text { DIRECTAMENTE } \\
\text { CON EL ALUMNADO } \\
\text { DIVERSO }\end{array}$} & Diagnósticos psicopedagógicos & & & & & \\
\hline & Seguimiento del alumnado con dificultades & & & & & \\
\hline & Elaboración de informes y pautas de intervención & & & & & \\
\hline & Diseñar actividades de refuerzo, recuperación, ... & & & & & \\
\hline & Diseño de RE y ACI & & & & & \\
\hline & Proporcionar información profesional y académica & & & & & \\
\hline & Aplicación de pruebas, tests, ... & & & & & \\
\hline & Actividades de acogida al alumnado nuevo & & & & & \\
\hline & Asesoramiento en el tratamiento de la diversidad & & & & & \\
\hline & Establecer medidas de prevención & & & & & \\
\hline & Facilitar pautas de actuación con alumnado diverso & & & & & \\
\hline & Asesorar y participar en la elaboración de proyectos & & & & & \\
\hline & Colaborar en el plan de acción tutorial & & & & & \\
\hline \multirow{8}{*}{$\begin{array}{l}\text { 32. EN QUE MEDIDA } \\
\text { IMPIDEN MEJORAR } \\
\text { LA DIVERSIDAD } \\
\text { EN TU CENTRO } \\
\text { LOS SIGUIENTES } \\
\text { OBSTÁCULOS }\end{array}$} & Bajo nivel conceptual del tema & & & & & \\
\hline & Falta de motivación en el profesorado & & & & & \\
\hline & Carencia de materiales didáctivos adecuados & & & & & \\
\hline & Ratios profesor/alumnado & & & & & \\
\hline & Falta de personal cualificado & & & & & \\
\hline & Falta de recursos (personales y materiales) & & & & & \\
\hline & Trabajo colaborativo & & & & & \\
\hline & Planificación adecuada & & & & & \\
\hline
\end{tabular}


Alonso, J. D., Castedo, A. L. \& Juste, M. P. (2013). Diseño y Propiedades Psicométricas de un Cuestionario de Opinión sobre la Atención a la Diversidad (CUADIVER).

\begin{tabular}{|c|c|c|c|c|c|c|}
\hline \multicolumn{2}{|c|}{ M (mala), R (regular), N (normal), B (buena), E (excelente) } & \multirow[t]{2}{*}{ M } & \multirow[t]{2}{*}{$\mathbf{R}$} & \multirow[t]{2}{*}{$\mathbf{N}$} & \multirow[t]{2}{*}{ B } & \multirow[t]{2}{*}{$\mathbf{E}$} \\
\hline \multirow{10}{*}{$\begin{array}{l}\text { 33. EN QUE MEDIDA } \\
\text { SE ENCUENTRA LA } \\
\text { ATENCIÓN A LA } \\
\text { DIVERSIDAD EN LOS } \\
\text { DOCUMENTOS DE TU } \\
\text { CENTRO }\end{array}$} & En el diseño curricular base (DCB) & & & & & \\
\hline & En el proyecto educativo de centro (PEC) & & & & & \\
\hline & En el proyecto curricular de centro (PCC) & & & & & \\
\hline & En las programaciones de aula & & & & & \\
\hline & En el plan de acción tutorial & & & & & \\
\hline & En el plan de orientación & & & & & \\
\hline & En los objetivos y contenidos didácticos & & & & & \\
\hline & En las actividades de enseñanza-aprendizaje & & & & & \\
\hline & En la metodología & & & & & \\
\hline & En la evaluación & & & & & \\
\hline \multirow{16}{*}{$\begin{array}{l}\text { 34. PARA MEJORAR } \\
\text { LA ATENCIÓN A LA } \\
\text { DIVERSIDAD EN } \\
\text { TU CENTRO SERIA } \\
\text { IMPORTANTE }\end{array}$} & Incrementar recursos humanos (especialistas ...) & & & & & \\
\hline & Incrementar recursos materiales (ordenadores ...) & & & & & \\
\hline & Mayor implicación administración educativa & & & & & \\
\hline & Actitudes positivas hacia la diversidad & & & & & \\
\hline & Potenciar la formación del profesorado & & & & & \\
\hline & $\begin{array}{l}\text { Aceptar al alumnado independientemente de su origen } \\
\text { étnico o cultural }\end{array}$ & & & & & \\
\hline & $\begin{array}{l}\text { Tener en cuenta los diferentes intereses y niveles del } \\
\text { alumnado (desterrar metodologías uniformes) }\end{array}$ & & & & & \\
\hline & $\begin{array}{l}\text { Romper el tradicional distanciamiento entre la } \\
\text { educación primaria y secundaria }\end{array}$ & & & & & \\
\hline & $\begin{array}{l}\text { Considerar positiva la composición diversa del } \\
\text { alumnado }\end{array}$ & & & & & \\
\hline & $\begin{array}{l}\text { Mayor autonomía en los centros educativos para } \\
\text { desarrollar y aplicar estrategias en la diversidad }\end{array}$ & & & & & \\
\hline & Acción tutorial & & & & & \\
\hline & Consolidación de equipos & & & & & \\
\hline & Mayor uso de estrategias curriculares & & & & & \\
\hline & Intervención y coordinación de los servicios & & & & & \\
\hline & Agrupamientos heterogéneos del alumnado & & & & & \\
\hline & $\begin{array}{l}\text { Flexibilizar la estructura y el funcionamiento del } \\
\text { centro para adaptarse a las diversas realidades }\end{array}$ & & & & & \\
\hline
\end{tabular}


Psicologia: Reflexão e Crítica, 26(2), 270-286.

\section{VALORACIÓN DE LAS MEDIDAS DE ATENCIÓN A LA DIVERSIDAD}

\{Valora del 1 al 5 en orden de importancia, las siguientes medidas de atención a la diversidad. Si desconoces alguna debes dejarla en blanco\}

\begin{tabular}{|c|c|c|c|c|c|}
\hline 1 (nada), 2 (poco), 3 (Suficiente), 4 (Bastante), 5 (mucho) & 1 & 2 & 3 & 4 & 5 \\
\hline 35. Medidas ordinarias a nivel de centro (PEC, PCC, PAT, ...) & & & & & \\
\hline 36. Medidas ordinarias a nivel de aula (Programaciones, materiales, ...) & & & & & \\
\hline 37. Acción tutorial y orientadora & & & & & \\
\hline 38. Refuerzos Educativos (RE) & & & & & \\
\hline 39. Agrupamientos flexibles & & & & & \\
\hline 40. Medidas ordinarias de atención a minorias étnicas (planes de acogida, ...) & & & & & \\
\hline 41. Permanencia o reducción de un año más en el ciclo & & & & & \\
\hline 42. Adaptaciones curriculares individualizadas (ACI) & & & & & \\
\hline 43. Medidas extraordinarias: acciones de atención a la diversidad cultural, $\ldots$ & & & & & \\
\hline 44. Otras (decir cuales) & & & & & \\
\hline
\end{tabular}

OBSERVACIONES: 